\title{
Nuevas localizaciones para Schenkia elegans (Samp.) Z. Díaz (Gentianaceae) en Andalucía Occidental con apuntes sobre su demografía
}

Zoila Díaz Lifante*, Carmen García Llamas, José Díaz Fernández \& Cristina Andrés Camacho

Departamento de Biología Vegetal y Ecología, Apdo. correos 1095, Avda. Reina Mercedes, Universidad de Sevilla, Sevilla, Spain

Correspondencia

Z. Díaz Lifante

e-mail: zoila@us.es

Recibido: 1 septiembre 2018

Aceptado: 30 septiembre 2018

Publicado on-line: noviembre 2018
New locations for Schenkia elegans (Samp.) Z. Díaz (Gentianaceae) in Western Andalusia with demographic notes

Palabras clave: Gentianaceae, flora, endemismo, conservación, corología, Península Ibérica, Andalucía, España.

Key words: Gentianaceae, flora, endemism, conservation, chorology, Iberian Peninsula, Andalusia, Spain.
Se confirma la presencia de esta especie singular de la tribu Chironieae (Gentianaceae), especie dada a conocer por primera vez en Flora Iberica (Díaz Lifante, 2012), con nuevas localizaciones de la provincia de Huelva, así como por primera vez en la provincia de Sevilla. Esto permite ampliar el conocimiento de su área de distribución, con carácter actualmente disyunto, y promover su búsqueda en otros lugares con hábitat similar para ratificar su carácter de especie endémica. Se ofrece información demográfica de estas nuevas localizaciones encaminada a plantear alguna medida de protección especial para la conservación de esta especie.

\section{Schenkia elegans (Samp.) Z. Díaz, Flora Iberica 11: 85 (2012)}

Centaurium spicatum raça elegans Samp., Man. Fl. Portug.: 383 (1913) [basiónimo]

Schenkia elegans fue descrita para el centro de Portugal, en la región histórica de Beira Litoral, que el autor había diferenciado de la raza típica por sus tallos más delgados, con ramas muy divergentes, y sus flores distantes y claramente pediceladas. Su morfología, en algunos rasgos común a Schenkia spicata (L.) G. Mans., como la presencia frecuente de cimas monocasiales y flores con el estigma subcapitado, permitió elevar la raça de Sampaio a categoría de especie bajo el mismo género. Plantas semejantes a éstas fueron encontradas en El Andévalo (Huelva) y en Aldeamayor de San Martín (Valladolid), como atestiguan varios pliegos de los herbarios de la Universidad de Sevilla (SEV) y de la Universidad de Salamanca (SALAF, incluido actualmente en SALA).
Esta especie es un terófito que forma poblaciones pequeñas y muy localizadas, coexistiendo a menudo junto a otras especies de la tribu Chironieae pertenecientes a los géneros Centaurium Hill, Schenkia Griseb. (S. spicata), Exaculum Caruel y Blackstonia Huds. Es posible encontrarla en pastizales efímeros desarrollados en los cauces desecados de corrientes de agua y en las márgenes secas de lagunas litorales y humedales de interior. Ocupa suelos de naturaleza preferiblemente ácidos, resultantes de la meteorización de esquistos y pizarras, pero también se encuentra en suelos algo salobres en zonas próximas al litoral.

Las únicas poblaciones conocidas hasta ahora, reflejadas en la distribución indicada en Flora Iberica, señalan a la especie como un endemismo ibérico, no habiéndose encontrado hasta la fecha ningún espécimen en los herbarios consultados que pudiese señalar lo contrario (ALME, BC, BCN, COI, FCO, G, GDA-GDAC, HUAL, JACA, JAEN, LISU, LISE, MA, MGC, MUB, SALA-SALAF, SANT, SEV, UNEX, VAL). Dentro de la Península Ibérica muestran una distribución disyunta, con presencia en el SO y NO de España (provincias de Huelva y Valladolid), y en el NO de Portugal (Beira Litoral).

Esta distribución está basada en los siguientes especímenes de herbario:

ESPAÑA. Huelva. Puebla de Guzmán, represa cercana al pueblo, 20-VII-1989, S. Silvestre (SEV-214292). Entre Tharsis y Villanueva de las Cruces, ladera próxima a depresiones húmedas, 37³6'37.6” - 75'35.3" O, 246 m, 6-VI-2002, Z. Díaz Lifante \& C. Santa-Bárbara (SEV-214423). Entre El Almendro y Alosno, depresiones secas 
próximas a cursos de agua, 37³1'36.7' $\mathrm{N}$ 7¹2'40.0" O, 202 m, 2-VII-2010, V. Girón \& Z. Díaz (SEV-249968). Valladolid. Aldeamayor de San Martín, 26-VII-1983, M. Ladero, F. Navarro \& C. Valle (SALAF-4797).

PORTUGAL. Beira Litoral. De Esmoriz a Mira, 30 June 1901, G. Sampaio (PO-Samp.).

La búsqueda en las mismas localidades donde anteriormente fue encontrada, tanto en la región portuguesa de Oporto como en la provincia española de Valladolid, así como en hábitats similares a los conocidos para la especie en otras zonas del oeste peninsular, ha sido infructuosa hasta el momento. Sin embargo se han producido nuevos hallazgos de la especie que suponen localizarla en dos regiones biogeográficas diferentes en Andalucía Occidental, dos de ellos nuevamente en la comarca de El Andévalo (Huelva), y otros tres, por primera vez, en la cuenca del bajo Guadalquivir (Sevilla).

Estas nuevas localizaciones para la especie son:

ESPAÑA. Huelva. Entre Puebla de Guzmán y El Almendro, bordes de arroyo próximo, 37³4'11.1" N - 7014'35.5" O, 207 m, 7-VI-2015, Z. Díaz Lifante (SEV-270205). Entre El Almendro y Alosno, proximidades a un curso de agua, 37 $31^{\prime} 12.3^{\prime \prime} \mathrm{N}$ - 7¹4'45.1" O, 236 m, 24-VI-2016, Z. Díaz Lifante \& C García Llamas (SEV 270231). Sevilla. Entre Sevilla y Montequinto, Campus de la Universidad Pablo de Olavide, 37²1'16.73" N - 556'15.57" O, 14 m, 1-VI-2015, Z. Díaz Lifante (SEV-2702013).
Coria del Río, Finca "Dehesa de la Atalaya", 37¹4'31.76" N - 559'43.55" O, 25 m, 5-VI-2016, Z. Díaz Lifante, C. Andrés, C. García Llamas \& J. Díaz Fernández (SEV-270230). Puebla del Río, "Dehesa de Abajo", 37¹2'31.64" N - 6¹0'45.50" O, 8 m, 19-6-2016, Z. Díaz Lifante, J. Díaz Fernández \& C. García Llamas (SEV-270229).

Un análisis demográfico efectuado en cuatro de las cinco poblaciones localizadas en junio de 2016, mediante transectos adecuados a la forma del área ocupada por la especie en la población estudiada, proporcionó información sobre la ecología, la densidad de población y los factores de amenaza, la cual se muestra en la tabla 1. La densidad de población encontrada en los muestreos fluctuó entre los 0.52 y 25.94 individuos por $\mathrm{m}^{2}$, en superficies siempre inferiores a $250 \mathrm{~m}^{2}$, lo que señala que la especie es poco abundante, aunque se necesita hacer más prospecciones para asegurar esto.

En la comarca de El Andévalo es probable que el área de distribución sea más extensa, dado que el hábitat ocupado por la especie es común en toda la zona, por lo que es esperable nuevas localizaciones en el futuro. A unos 3 km de la población muestreada existía en 2010 una población muy extensa (representada por el espécimen de herbario SEV-249968), que ocupaba los bordes y cauce seco de un arroyo, la cual sin embargo desapareció en el año 2016 por obras amplias de roturación de los márgenes

\begin{tabular}{|c|c|c|c|c|}
\hline Población y testigo & Ecología & $\begin{array}{l}\text { Superficie de } \\
\text { muestreo }\left(\mathrm{m}^{2}\right)\end{array}$ & $\begin{array}{c}\mathrm{N}^{\circ} \text { de } \\
\text { individuos }\end{array}$ & Grado de amenaza \\
\hline $\begin{array}{l}\text { Entre El Almendro } \\
\text { y Alosno. } \\
\text { SEV-270231 }\end{array}$ & $\begin{array}{l}\text { Bordes de arroyo, en claros de } \\
\text { vegetación, sobre suelos ácidos de } \\
\text { pizarras y esquistos. }\end{array}$ & $\begin{array}{l}34 \\
78\end{array}$ & $\begin{array}{l}882 \\
217\end{array}$ & $\begin{array}{l}\text { Proximidad de caminos y } \\
\text { campos cultivados. }\end{array}$ \\
\hline $\begin{array}{l}\text { Campus de la } \\
\text { Universidad Pablo } \\
\text { de Olavide. } \\
\text { SEV-270213 }\end{array}$ & $\begin{array}{l}\text { Parcela ajardinada, sobre suelo } \\
\text { aluvial arcilloso, ligeramente } \\
\text { ácido y salino, encharcado } \\
\text { estacionalmente. }\end{array}$ & 218.88 & 1488 & $\begin{array}{l}\text { Parcela protegida, pero } \\
\text { anualmente segada, } \\
\text { antes de que fructifique la } \\
\text { especie. }\end{array}$ \\
\hline $\begin{array}{l}\text { Dehesa de Abajo. } \\
\text { SEV-270229 }\end{array}$ & $\begin{array}{l}\text { Bordes de arroyos estacionales } \\
\text { en claros de pinar-acebuchal, } \\
\text { sobre suelos ácidos con arena y } \\
\text { conglomerados. }\end{array}$ & 675 & 536 & $\begin{array}{l}\text { Bajo riesgo al situarse } \\
\text { en la "Reserva Natural } \\
\text { Concertada Dehesa de } \\
\text { Abajo" }\end{array}$ \\
\hline $\begin{array}{l}\text { Dehesa de la } \\
\text { Atalaya. } \\
\text { SEV-270230 }\end{array}$ & $\begin{array}{l}\text { Bordes de lagunas estacionales, } \\
\text { en claros de pinar-acebuchal, } \\
\text { sobre suelos ácidos con arena y } \\
\text { conglomerados. }\end{array}$ & $\begin{array}{l}46.35 \\
247.59 \\
118.80\end{array}$ & $\begin{array}{c}24 \\
159 \\
336\end{array}$ & $\begin{array}{l}\text { Cierto riesgo al estar en } \\
\text { zona catalogada como } \\
\text { "Área forestal de interés } \\
\text { recreativo" }\end{array}$ \\
\hline
\end{tabular}

Tabla 1: Características ecológicas, demografía y grado de amenaza actual de las poblaciones de Schenkia elegans muestreadas en 2016.

Table 1: Ecology, demographic information and threat level of the populations of Schenkia elegans sampled in 2016. 
de la carretera, extendidas a más de 20 metros de la cuneta. La población situada en el Campus de la Universidad Pablo de Olavide (Sevilla) es muy singular, pudiendo quizás explicarse su presencia allí por la dispersión a través de las aves que frecuentan estos lugares temporalmente encharcados, derivados, según Luceño et al. (2005), de las capas de aluviones de las antiguas terrazas del río Guadaíra. Deben tomarse medidas encaminadas a preservar el banco de semillas de la especie, mediante el retraso de la siega de la parcela a fechas posteriores al mes de junio, en que fructifica la especie. La población Dehesa de Abajo está muy localizada dentro del paraje conocido como Reserva Natural Concertada Dehesa de Abajo, próxima al Espacio Natural de Doñana. Es una zona de gran interés florístico, al estar en una zona de contacto entre la Comarca de El Aljarafe, las marismas del Guadalquivir y los arrozales de Isla Mayor.

La población más extensa se encuentra en la Dehesa de la Atalaya, formada por varias subpoblaciones. Este paraje constituye una "finca de propios" del pueblo de Coria del Río, asentada en la margen izquierda del río Guadalquivir. La mayor parte de la finca está ocupada por zonas cultivadas, pero hay una extensa zona forestal de acebuchal, pinar y algunos ejemplares de encinas. Existen en la zona pequeñas lagunas estacionales, originadas por la antigua extracción de áridos, las cuales se han naturalizado y albergan hoy día ecosistemas interesantes de cierta riqueza biológica. Esto favorece el ecoturismo en esta zona, lo que puede suponer un factor futuro de amenaza. Otro factor de amenaza podrían ser los cambios en los planes de explotación de la finca para la agricultura o la aparición de factores que determinen la desecación de las lagunas, como drenajes incontrolados.

Las nuevas localizaciones señalan que el área de distribución de la especie podría ser más extensa de lo que sus hallazgos parecen indicar. Probablemente su pequeño tamaño y su gran parecido con otras gencianáceas de flor pequeña, como $S$. spicata, Centaurium tenuiflorum (Hoffmanns. \& Link) Fritsch y C. pulchellum (Sw.) Druce, hayan determinado que pasase desapercibida hasta ahora. El descubrimiento de nuevas poblaciones permitirá ratificar y ampliar las características ecológicas de la especie, lo que podría traducirse en nuevos hallazgos que mejoren el conocimiento de su rareza, en aras a tomar medidas de protección especial. Por el momento los estudios demográficos aquí mostrados indican un área de ocupación de las poblaciones pequeño, con un número de individuos inferior a 10.000. Esto, unido al escaso número de poblaciones conocidas para la especie, representa valores propios de especies vulnerables. Pero es necesario acometer más estudios sobre su distribución geográfica, demografía y grado de amenaza para ratificar su status de conservación, quedando la especie pendiente de evaluación para la catalogación en alguna figura de protección contemplada por la legislación vigente. Por tanto, con los conocimientos actuales y según las Categorías de amenaza de la UICN (UICN, 2001), la especie se debería considerar en la categoría de especie "No Evaluada" (NE). No obstante, hasta disponer de la información suficiente esta especie debería protegerse del mismo modo que lo están otras especies amenazadas.

\section{Agradecimientos}

Los autores están muy agradecidos a C. SantaBárbara por su colaboración en las primeras incursiones por la comarca de El Andévalo, así como a otras personas que nos acompañaron en la búsqueda de la especie, y a la Agencia de Medio Ambiente de Coria del Río (Sevilla), la cual facilitó el acceso a la Dehesa de la Atalaya. La excursión efectuada en 2016 a Beira Litoral y Valladolid para buscar la especie fue financiada por el proyecto MINECO (CGL2013-45037-P), dirigido por el Prof. J. Arroyo.

\section{Bibliografía}

Díaz Lifante, Z. (2012). Schenkia Griseb. In S. Castroviejo et al. (Eds.), Flora Iberica, 11 (pp. 81-86), Madrid, CSIC.

Luceño, M., Jiménez, P., Escudero, M., Martín, S., Narbona, E. (2005). Flora silvestre y ornamental del campus de la Universidad Pablo de Olavide. Consejería de Medio Ambiente de la Junta de Andalucía - Universidad Pablo de Olavide. Sevilla.

Sampaio, G.A. (1913). Manual da flora portuguesa. Tipografia Occidental, Porto.

UICN (2012). Categorías y Criterios de la Lista Roja de la UICN: Versión 3.1, 2 ed. Comisión de Supervivencia de Especies de la UICN. UICN, Gland, Suiza, y Cambridge, Reino Unido. 